\title{
Adaptive Case Management in the Social Enterprise
}

\author{
Hamid Reza Motahari Nezhad, Claudio Bartolini, Sven Graupner, and Susan Spence
}

\author{
Services Research Lab, Hewlett Packard Laboratories \\ Palo Alto, California, USA \\ \{hamid.motahari, claudio.bartolini, sven.graupner, \\ susan. spence\} ahp.com
}

\begin{abstract}
In this paper, we introduce SoCaM, a framework for supporting case management in social networking environments. SoCaM makes case entities (cases, processes, artifacts, etc.) first class, active elements in the social network and connects them to people. It enables social, collaborative and flexible definition, adaptation and enactment of case processes among people. It also offers mechanisms for capturing and formalizing feedback, from interactions in the social network, into the case, process and artifact definitions. We report on the implementation and a case management scenario for sales processes in the enterprise.
\end{abstract}

Keywords: Case Management, Ad-hoc Processes, Enterprise Social Networks.

\section{Introduction}

Case management accounts for a large proportion (>90\%) of process-centric activities in the enterprise, where knowledge workers are involved in domains such as customer relationship management, IT service management, health, legal, financial, government and telecommunications [4]. A case consists of a set of artifacts, actors (human or systems), tasks - planned and unplanned - and the coordination of the tasks to achieve a certain goal [5]. Adaptive case management (ACM) refers to managing all of the work needed to handle a specific case in a flexible manner by adhering to the principles of planning-by-doing and accommodating changes in the environment [3, 4]. Managing cases often involves a mix of automated work (emails, communication, document and partly workflow management applications) and human work. The main inefficiency in today's case management comes from the fact that the people, information systems, process definitions and enactment tools are isolated and disconnected.

Social media and in particular social networks are emerging as the main communication and interaction platforms for people. Social networks have been deployed in the enterprise (e.g., platforms such as Yammer, Jive Engage, SocialText, Salesforce Chatter, and HP WaterCooler ${ }^{1}$ ). However, their use is limited to information sharing among employees and they have not been linked to and used as

\footnotetext{
${ }^{1}$ WaterCooler is an internal HP social networking platform [6].
} 
productivity tools in the enterprise. This is reflected in their low adoption in the enterprise. For example, less than $5 \%$ of employees are active users of HP WaterCooler $[6,14]$ even though it has been deployed in HP for more than five years.

In this paper, we present SoCaM, a framework for flexible case management centered on an extended notion of an enterprise social network that consists of people and things. The main contributions are as follows:

- We define process, task and artifact as first class entities in the social network of people and things.

- We define a case as a first class entity in the social network. A case may include one or more processes as the basis for the enactment of the case.

- We support the adaptive enactment of best practice processes for case management applications by offering a wide range of flexibility features including adaptive tasks, adaptive case templates and partially fulfilled tasks, and the addition of at-will ECA (event-condition-action) rules.

- We enable process, task and artifact to become active entities to subscribe to a case that uses them, so they are informed about and can identify the changes made to their definitions during case enactment.

- We report on a use case of this technology in the domain of sales processes in the HP Enterprise Services organization.

The paper is structured as follows. In Section 2, we present the requirements for adaptive case management in a social context. In Section 3, we present SoCaM, the proposed framework for social case management. Section 4 presents the architecture, the implementation of a prototype SoCaM framework and the case study. We discuss related work in Section 5 and conclude with future work in Section 6.

\section{Requirements of $\mathrm{ACM}$ in the Social Enterprise}

We conducted a case study on sales case management within the HP Enterprise Services business. We focused on how people in a sales team collaborate within and across teams, and how they define, enact and coordinate their activities for a sales case. We found that there are best-practice process definitions that provide guidelines on the sales process. Sales teams decide on the actual activities that they perform in a specific sales case. A key observation is that the work is defined around artifacts of the case. Tasks define work stages on the artifacts, what input(s) are needed and what output(s) are generated. The case may use one or more process templates, and tasks in the case either belong to a process template or are added in an ad-hoc manner. There is a need for flexibility in task execution order. Many of the tasks are optional by definition. Many of the tasks may be completed after several rounds of execution (so there is a need for support for partial fulfillment) where, in each round, the tasks work on the same set of artifacts as input/output. Finally, process definition configurability is important for tasks and templates to enable the process to be adapted for the case as more contextual information on the case becomes available.

Adding the perspective of an enterprise social network, we see the need for making process, artifact and task active in the social network so that, like other 
entities in the network (such as people), they should also be capable of emitting and consuming events. Beyond representation, the ability for these entities to subscribe to information about their usage and to analyze feedback is also important. Entities can use such information to enhance their representation and to adjust their behavior in the network (in terms of the events that they emit).

\section{SoCaM: The Social Case Management Framework}

The Social Case Management (SoCaM) framework is designed for the definition and enactment of best practice processes in an enterprise social network environment. SoCaM represents process, task, artifact and case as first class entities in the social network. In this approach, each best practice process is registered in the network environment. Each individual task of a process may be also registered as a first class entity. This enables the sharing of reusable tasks across several processes, and also the receiving of finer granularity feedback from the network at the task level. The profile of the task includes a description of how the task needs to be performed, the roles that are involved in the task, input and output artifacts, task variances (based on configurable parameters such as deal type, size, geography and sales region), and the list of supporting resources. The process (template) profile then includes a description, a list of tasks and the precedence of tasks which can be represented as a dependency graph or a list view.

When a new case is created in the SoCaM environment, the case manager may choose a number of configuration parameters. In the context of the Services Sales domain, the configurable parameters are: region, industry, size of the deal, and deal type. SoCaM prepares the list of compatible processes and a recommended set of process templates are presented to the user. The user may choose to include one or more of the process templates in the case. Examples of process templates are pursuit management, solution management, customer management, etc. These will be added to the task space of the cases, using each process as a way to group tasks. Note that tasks in the added process templates may have dependencies (based on their input/output). These dependencies will be automatically established by the SoCaM engine. The user may define additional precedence constraints on the tasks across templates.

\subsection{The SoCaM Data Model}

The SoCaM data model is depicted in Fig. 1. A case consists of a set of tasks and a set of artifacts. A case may be associated with one or more process definition templates. A process (template) represents the definition of a best practice in the organization. A process consists of a set of inter-related tasks. The task relationships capture their recommended precedence. Artifacts are documents and they are first class entities in case management. We define an artifact template such that an artifact is an instance of a template. An artifact template is independent of any case, while an artifact, on the other hand, is an instantiation of a template for a specific case. 


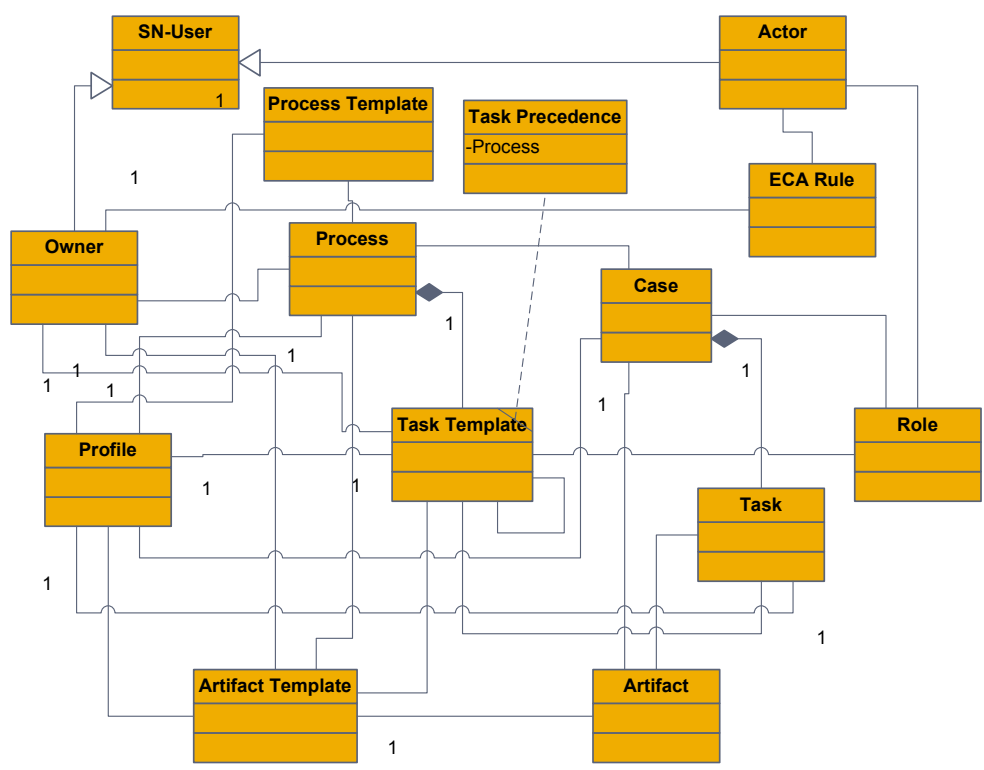

Fig. 1. The conceptual data model for representing case entities in a social network environment.

Task. A task has a profile, an owner, a set of attributes, including state, and a set of roles associated with it. Having a profile means that the task can be followed by the social network users. We represent a task template as a tuple $t=<$ name, state, roles, artifacts-templates, permission, owner, followers $>$. The state $=<$ Ready, Assigned, Pending, Completed $>$, and this list can be updated by the task owner. The possible set of roles $=<$ Accountable/Approver, Responsible, Follower $>$. An actor may be assigned as Accountable/Approver (as somebody who is eventually accountable, and optionally can be mandated to approve the task before it is declared as completed), Responsible (the person who performs the task), or Follower (somebody, assigned at execution time, who is interested in being informed of the status of the task during execution in a case). The followers list, on the other hand, refers to network users who follow updates to the definition of this task. The owner is a user in the social network who has edit authority on the task. The permission specifies the view and edit access permissions on the task. By default, the view access is set to case actors, and the edit access is set to responsible/accountable actors. These permission settings and those for comments/updates on the task can be expanded to include followers, all social network users or specific external users (through their email). We use the term task to refer to the instantiation of a template. A task has a set of actors associated with it.

Process. A process is composed of a set of tasks that are inter-related. We define a process template as $p t=<$ name, tasks, tpgraph, artifact-templates, owner, permission, followers $>$. The variable tpgraph refers to the task precedence graph, which is a graph that shows the recommended relationships among tasks, based on the dependencies among their input/outputs. The tpgraph may represent a multi-level graph for a process in which some tasks may include a tpgraph for the set of their subtasks. We use term process to refer to the instantiation of a template for a specific case. 
Case. A case is a container for a collection of adaptive tasks performed on a set of artifacts to achieve a certain goal, e.g., to handle a specific service engagement. A case is defined as $c=<$ name, processes, tasks, artifacts, actors, permission, followers $>$. A case contains one or more processes (and references to associated process template references) which are enacted during the course of case handling. The social network users that are involved in the case are called actors, and may be assigned to one of an extensible list of roles in the case. The default role is the casemanager who is ultimately accountable for the case. A case may have a number of followers, who receive public updates from the case. An actor is a social network user who takes a role in a case or in a specific task. An actor (and also an owner, as a social network user) may define a number of ad-hoc (Event-Condition-Action) rules on events related to cases, tasks, processes and artifacts.

\subsection{Flexible Process Enactment for Case Management}

The SoCaM case management engine adopts the WS-HumanTask framework [12] for the definition of human tasks. The relationships between tasks in the context of a case are modeled using a dependency graph that maintains the relationships between cases, actors, tasks, artifacts and processes. This graph can dynamically change during the course of case handling. In addition to the flexibility features such as adding a task, skipping a task, and removing a task, we also support:

Adaptive tasks: tasks that adapt to the context of the case. The model allows parametric modeling and configuration of tasks, that enables them to be enacted differently in different contexts; for example, Service deals may be characterized by parameters such as the size of the deal, the solution complexity, and different geographical regions.

Adaptive process templates: The system adapts the order and number of tasks in a case process template based on changes in the contextual parameters of the case.

Partially fulfilled tasks: identifying tasks that can be partially fulfilled and completed during several rounds of execution, often based on the acceptance/review conditions. The important aspect is that, in each iteration, the task will act on the same artifacts.

Social tasks: tasks that are open to all social network users or selected external users through permission settings and get completed based on some conditions such as minimum number of contributions, etc. This enables the soliciting of contributions to the case through crowdsourcing practices, by engaging all enterprise employees, or from external users such as customers.

\subsection{Supporting Cases in a Social Network}

The case profile names it, describes its purpose, presents its current status and provides pointers to related entities including people and tasks. A case profile is a container and aggregator of activities around the case, and these activities are posted to the activity pages of actors and followers of the case. Fig. 2 shows a sample case 


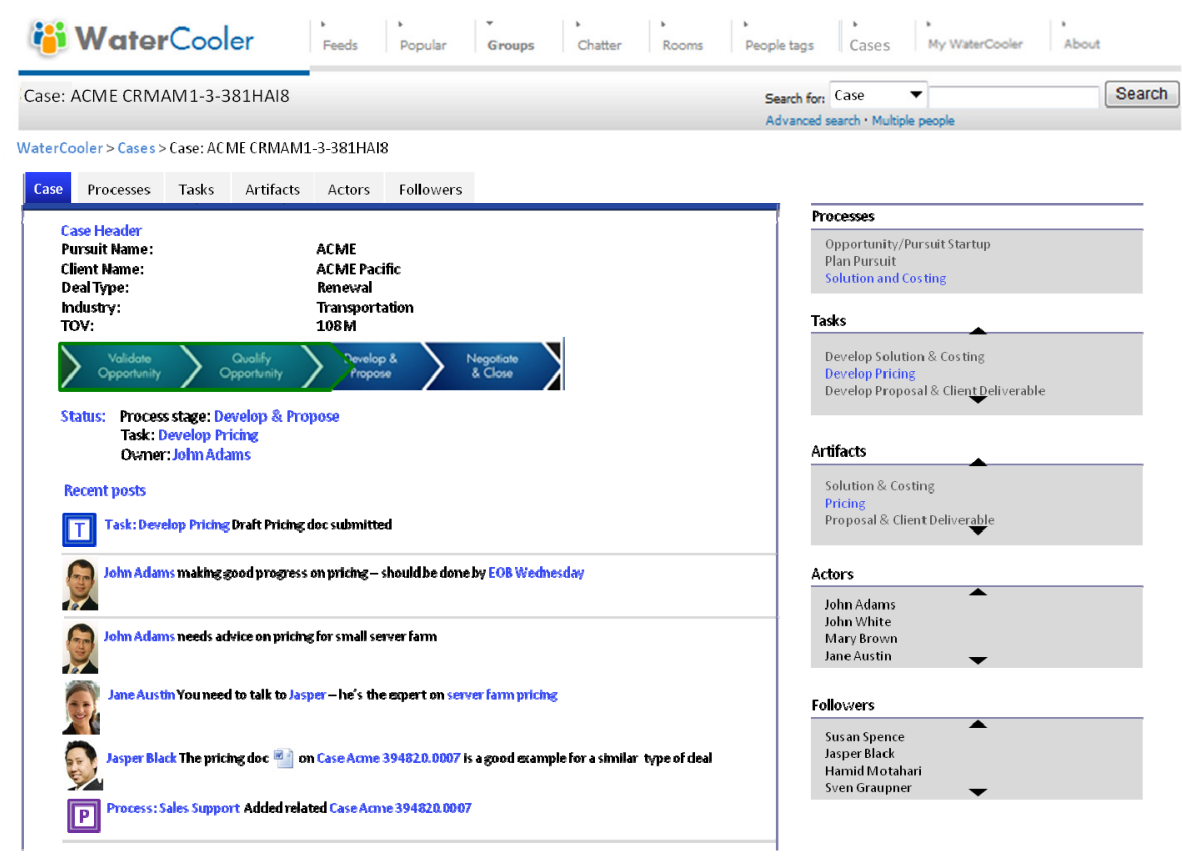

Fig. 2. The case elements in a social network environment

profile in HP WaterCooler. A social network user can explore the description and composition of the case to see what best practice guidelines it enacts, and which process templates it uses, and view the current status of the case and choose to follow the case.

A social network user who is a case actor can also view a list of past and current activities on the case, tailored to their role, participate in discussions by commenting e.g. associated with a process or task, to justify an action such as skipping a task, which will both form a part of the record for this case history and be reflected back to the process usage history in the process template. Each case has an email address. Users can include the case in the email exchanges. The emails are posted to the case profile on behalf of the sender. This feature addresses information lock-in among enterprise employees since, today, a lot of information related to the case or individual tasks are locked in emails, or spread across multiple communication channels such as email and IM.

\subsection{Implementation and Case Study}

We have developed SoCaM on top of HP WaterCooler (see [14]). We extended the data model of WaterCooler by creating nodes for entities (process, task, case and artifact) and related information in a graph database. For the data model, we have extended Neo4J (neo4j.org/), a scalable graph database, to represent and store information about cases, and to relate cases to people and other entities in WaterCooler. The template manager module supports the definition of process, task 
and artifact templates and maps them into the graph node representation. The task definitions are based on the WS-HumanTask framework implementation from jBoss (jbpm.org). The rule definitions are mapped into the jBPM Drools framework. We plan to use a distributed publish/subscribe model such as PADRES [13] (padres.msrg.org) that suits the social network environment.

As a case study, we have used SoCaM in the context of case management for sales cases. We modeled the sales processes which include hierarchical, multi-level process definitions as process templates in SoCaM, and their tasks as task templates. Many sales people were already familiar with WaterCooler but their use of it was limited to sharing information. We received very supportive and encouraging feedback on how this framework facilitates the job of sales people, and addresses the major issues related to information silos, broken and fragmented communications, and the need for transparent and flexible case management in social network environments.

\section{$4 \quad$ Related Work}

We study related work in three categories: adaptive case management, artifact-centric process management, and business process management in social networks.

Adaptive case management. Case management is a hot topic in research and practice today $[1,2]$, and in academia it has been studied in various contexts including health $[3,4,5]$. In SoCaM, we adopt the same basic principles as in [3] and could use a similar formal framework to formally describe our model. The main advances include that our framework supports extra flexibility features such adaptive tasks, adaptive templates and social tasks for opening up tasks to actors outside the system, and also enables actors to define ad-hoc personal rules to manage the case.

Artifact-centric business processes. The artifact-centric approach for business processes represents a class of approaches [9, 10] in which the state changes and evolution of business artifacts are considered as the main driver of the business process. While we share the same observation that business artifacts are the center of attention in case management applications, we take a hybrid approach in SoCaM where object lifecycle (as input or outcome of tasks or cases) is important, but the model contains tasks that define activities over artifacts and some control flow level actions to take the case forward.

Social BPM. Recently the topic of social BPM has received a lot of interest in academia and practice [7, 8]. Most approaches by BPM vendors or research offer extensions for business process models to represent tasks that can be exposed in social networks (e.g., for voting, ranking, etc). [7] offers a model driven approach for generating code for tasks in popular social networks such as Facebook and LinkedIn and also an extended business process management engine. Current effort focuses on supporting traditional workflow applications in the social network environment. We go beyond and support the enactment of ad-hoc processes in the context of adaptive case management in enterprise social networks.

\section{$5 \quad$ Conclusions and Future Work}

We have presented SoCaM, a framework for social case management. We provide a technology solution for flexible case management in the social network environment 
that represents case elements as first class entities in the social network, and includes provision for collecting and analyzing feedback about the usage of process, task and artifact entities. The benefits of case management using SoCaM include the ease of capturing and enabling the exploration of relationships between people and entities, offering a context for sharing and keeping a record of knowledge about cases, processes, tasks and artifacts and also the ability to use notifications, based on a publish/subscribe model, to communicate changes and updates. Research to support case management applications in a social network environment is in its early stages. We plan to make our research prototype more scalable in a production environment, specifically w.r.t. distributing and propagating changes and subscribing to events.

\section{References}

1. Singularity, Case Management: Combining Knowledge with Process (July 2009), http: / / www. bptrends.com/publicationfiles / 07-09-WP-CaseMgtCombiningKnowledgeProcess-White.doc-final.pdf

2. de Man, H.: Case Management: A Review of Modeling Approaches (January 2009), http: / / www. bptrends. com/publicationfiles/01-09-ART\%20 Case 20 Management-1-DeMan. $\% 20$ doc-final.pdf

3. van der Aalst, W.M.P., Weske, M., Grünbauer, D.: Case handling: a new paradigm for business process support. Data Knowl. Eng. 53(2), 129-162 (2005)

4. Burns, E.V.: Case Management 101. In: Swenson, K.D., Palmer, N., Silver, B., Fischer, L. (eds.) Taming the Unpredictable. Future Strategies Inc. (2011)

5. Palmer, N.: BPM and ACM (Adaptive Case Management). In: Swenson, K.D., Palmer, N., Silver, B., Fischer, L. (eds.) Taming the Unpredictable. Future Strategies Inc. (August 2011)

6. Brzozowski, M.: WaterCooler: Exploring an Organization Through Enterprise Social Media. In: Proc. the ACM International Conference on Supporting Group Work, pp. 219228 (2009)

7. Fraternali, P., Brambilla, M., Vaca, C.: A Model-driven Approach to Social BPM Applications. In: Fischer, L. (ed.) Social BPM. Future Strategies Inc. (May 2011)

8. Fischer, L.: Social BPM. Future Strategies Inc. (May 2011)

9. Kumaran, S., Liu, R., Wu, F.Y.: On the Duality of Information-Centric and ActivityCentric Models of Business Processes. In: Bellahsène, Z., Léonard, M. (eds.) CAiSE 2008. LNCS, vol. 5074, pp. 32-47. Springer, Heidelberg (2008)

10. Bhattacharya, K., Gerede, C., Hull, R., Liu, R., Su, J.: Towards Formal Analysis of Artifact-Centric Business Process Models. In: Alonso, G., Dadam, P., Rosemann, M. (eds.) BPM 2007. LNCS, vol. 4714, pp. 288-304. Springer, Heidelberg (2007)

11. Salesforce, Sales process management and workflow (2012), http: //www. salesforce.com/crm/sales-force-automation/workflow/

12. OASIS, Web Services - Human Task (WS-HumanTask) Specification Version 1.1 (November 2009), http: / / docs . oasis-open. org/bpel4people/ ws-humantask-1.1-spec-cd-06.pdf

13. Wun, A., Jacobsen, H.-A.: Modelling performance optimizations for content-based publish/subscribe. In: Proceedings of the 2007 Inaugural International Conference on Distributed Event-Based Systems (DEBS 2007), pp. 171-179. ACM, USA (2007)

14. Graupner, S., Bartolini, C., Motahari-Nezhad, H., Mirylenka, D.: Social Media Meet the Enterprise - Analysis, Conclusions and Advancing to the Next Level. In: The Proceedings of EDOC 2012, China (September 2012) 